\title{
Associação entre religiosidade, atividade física e comportamento sedentário em adolescentes
}

\author{
Association between religiosity, physical \\ activity, and sedentary behavior in \\ adolescents
}

Rev Bras Ativ Fis Saúde p. 359-369

DOI: http://dx.doi.org/10.12820/2317-

1634.2012v17n5p359

1 Universidade de Pernambuco. Programa Associado de Pós-graduação em Educação Física UPE/UFPB. Programa de Pósgraduação em Hebiatria. Recife, Brasil.

2 Grupo de Pesquisa em Estilos de Vida e Saúde

3 Instituto Federal de Educação de Sergipe, Aracajú, SE, Brasil.

4 Universidade Federal de Alagoas, Maceió, AL, Brasil.

\author{
Edilânea Nunes Mélo ${ }^{1,2}$ \\ Aldemir Smith Meneses ${ }^{3}$ \\ Agostinho Gonçalves da Silva Júnior ${ }^{4}$ \\ Rildo de Souza Wanderley Júnior ${ }^{1}$ \\ Mauro Virgílio Gomes de Barros ${ }^{4}$
}

\section{Resumo}

O objetivo deste estudo transversal foi analisar a associação da religiosidade com o nível de atividade física, comportamento sedentário e participação na educação física em adolescentes estudantes do ensino médio da rede pública do estado de Pernambuco. Dados de 4207 estudantes (14-19 anos) foram coletados mediante utilização do Global School-based Health Survey. Os dados foram analisados mediante análises bivariáveis e multivariáveis. Comparados aos adolescentes que relataram "não ter religião", os católicos tinham maior chance de apresentar nível insuficiente de prática de atividade física $(\mathrm{OR}=1,39$; IC95\%:1,14-1,71) e comportamento sedentário em dias do final de semana ( $\mathrm{OR}=1,26$; IC95\%:1,03-1,53). Identificou-se também que os evangélicos apresentaram chance 76\% superior de não participar das aulas de educação física (OR=1,76; IC95\%:1,36-2,27). A prática religiosa estava associada a todas as variáveis, exceto à atividade física. Concluiu-se que a religiosidade é um fator associado à atividade física, comportamento sedentário e participação na educação física.

\section{Palavras-chave}

Religião; Comportamento do adolescente; Atividade motora; Estilo de vida sedentário.

\begin{abstract}
The aim of this cross-sectional study was to analyze the association between religiosity and physical activity level, sedentary behavior and enrollment in physical education classes among adolescent attended in public high school from Pernambuco State. Data from 4,207 students (14-19 years old) were obtained using the Global School-based Health Survey. Multiple and bivariate analyzes were performed. The adolescents who reported being Catholic were more likely to be insufficiently active $(O R=1.39 ; 95 \% C I: 1.14,1.71)$ and exposed to sedentary behavior during weekend days $(O R=1.26$; 95\%CI: 1.03, 1.53) in comparison to those who reported 'no religious affiliation'. In addition, Protestants showed $76 \%$ higher likelihood of no enrollment in physical education classes $(O R=1.76 ; 95 \% C I: 1.36,2.27)$. The religious practice was a factor associated to all dependent variables, except for the physical activity level. These results support that religiosity was associated with physical activity, sedentary behavior and enrollment in physical education classes.
\end{abstract}

\section{Keywords}

Religion; Adolescent behavior; Motor activity; Sedentary lifestyle.

Você pode ter acesso a este artigo na sua 


\section{INTRODUÇÃO}

Os benefícios da prática de atividades físicas para saúde estão bem documentados na literatura ${ }^{1}$. Apesar disso, estudos mostram elevada prevalência de níveis insuficientes de prática de atividades físicas tanto em adolescentes brasileiros ${ }^{2}$ quanto de outros países ${ }^{3}$, particularmente entre as moças e entre os jovens com maior idade 4,5 . As evidências disponíveis sugerem também a existência de uma tendência de declínio do nível de atividade física com o aumento da idade que se expressa de maneira mais pronunciada durante a adolescência ${ }^{6,7}$.

Paralelamente à redução do nível de atividade física, observa-se também que a população jovem está mais exposta aos chamados comportamentos sedentários ${ }^{7}$ e a menor frequência de participação nas aulas de educação física ${ }^{8}$. $\mathrm{O}$ aumento na prevalência de exposição a estes fatores comportamentais de risco, isolados ou em combinação, podem estar entre as causas para elevada incidência de excesso de peso em populações jovens e, mais tardiamente, para crescente incidência de obesidade e doenças cardiocirculatórias em adultos ${ }^{9,10}$.

Uma linha de investigação que vem sendo adotada para estudar as razões para a crescente prevalência de níveis insuficientes de atividade física e comportamentos sedentários é a identificação de fatores associados. Há também estudos desenvolvidos com o objetivo de analisar estratégias visando a promoção da atividade física, como as investigações sobre o papel que as aulas de educação física podem assumir no aumento do nível de atividade física dos jovens ${ }^{11,12}$.

Como observado em relação a outros eventos de saúde, uma hipótese que vem sendo discutida é de que a religião pode ser um fator associado à prática de atividades físicas e comportamentos sedentários porque fatores religiosos podem exercer influência no desenvolvimento de atitudes, crenças e valores das pessoas, mesmo entre as mais jovens. Quando uma pessoa se vincula a uma denominação religiosa, ele está aderindo, também, a um conjunto de símbolos, comportamentos e práticas sociais que podem modular o seu estilo de vida e, por conseguinte, afetar sua saúde ${ }^{13}$.

Apesar de algumas doutrinas religiosas conduzirem os seus praticantes a adotarem condutas de risco à saúde ${ }^{14,15}$, de um modo geral, os estudos sugerem existir uma associação positiva entre a religiosidade e as práticas de saúde ${ }^{16-18}$. Há evidências de que a prática religiosa está positivamente associada ao bem-estar físico ${ }^{19}$ e mental $^{16}$. Além disso, nota-se que a falta de uma prática religiosa efetiva está associada à adoção de diversas condutas de risco à saúde, tais como tabagismo, etilismo, uso de drogas ilícitas e comportamento sexual de risco ${ }^{20,21}$.

Em relação às atividades físicas e comportamentos sedentários, estudos com adultos evidenciaram que indicadores de religiosidade (participação em eventos religiosos, compromisso religioso, apoio social divino) são fatores associados a maiores níveis de atividade física ${ }^{22,23}$. Estudos com adolescentes são escassos, mas é possível levantar a hipótese de que a religiosidade esteja associada ao nível de atividade física em pessoas jovens. Assim, o objetivo deste estudo foi analisar a associação de indicadores de religiosidade (afiliação e prática religiosa) com o nível de atividade física, comportamento sedentário e participação em aulas de educação física em adolescentes estudantes do ensino médio.

\section{MÉTOdOS}

Para desenvolvimento do estudo foi adotado delineamento transversal, de base- 
escolar e abrangência estadual (Pernambuco, Brasil). Trata-se de estudo realizado a partir de análise secundária de dados do projeto intitulado "Estilos de Vida e comportamentos de Risco à Saúde em Adolescentes: do estudo de prevalência à intervenção". O protocolo de investigação foi submetido e aprovado pelo Comitê de Ética em Pesquisa com Seres Humanos em atendimento às normas estabelecidas nas Resoluções 196 e 251, do Conselho Nacional de Saúde.

A população alvo - estimada em 352.829 sujeitos segundo dados da Secretaria da Educação e Cultura do Estado de Pernambuco - foi constituída por estudantes do ensino médio matriculados na rede pública estadual, com idade entre $14 \mathrm{e}$ 19 anos. Para cálculo do tamanho da amostra foram adotados os seguintes parâmetros: intervalo de confiança de 95\%; erro máximo tolerável de 3\%; efeito de delineamento amostral de 4; e, prevalência estimada em 50\% (opção adotada em função dos múltiplos fatores analisados no estudo). A partir destes parâmetros o tamanho amostral calculado foi de 4217 sujeitos.

Do ponto de vista do processo amostral, procurou-se garantir que os estudantes selecionados representassem a população alvo quanto à distribuição conforme região geográfica, tamanho da escola e período de matrícula (diurno/noturno). A distribuição regional foi analisada a partir do número de estudantes matriculados em cada uma das 17 GEREs (Gerências Regionais de Ensino). O tamanho da escola foi classificado em três níveis de acordo com o número de alunos matriculados no ensino médio (rede pública), observando-se os seguintes critérios: porte I (pequeno) - menos de 200 alunos; porte II (médio) - 200 a 499 alunos; e porte III (grande) - 500 alunos ou mais. Os alunos matriculados nos períodos da manhã e da tarde foram agrupados numa única categoria (estudantes do período diurno). Todos os estudantes das turmas sorteadas foram convidados a participar do estudo.

A seleção da amostra foi realizada mediante amostragem por conglomerados em dois estágios, adotando-se a escola e turma como unidade amostral primária e secundária, respectivamente. No primeiro estágio, procedeu-se ao sorteio das escolas, de modo a incluir no mínimo uma escola de cada porte por GERE. No segundo estágio, foram sorteadas 203 turmas entre aquelas existentes nas escolas sorteadas no primeiro estágio para que o tamanho amostral desejado pudesse ser alcançado.

O instrumento utilizado na coleta dos dados foi o questionário Global Schoolbased Student Health Survey (GSHS), proposto pela Organização Mundial de Saúde com o objetivo de avaliar a exposição aos comportamentos de risco à saúde em adolescentes. A versão em português deste instrumento foi submetida a processo de validação e estudo piloto e está disponível aos leitores por solicitação aos autores deste manuscrito. $\mathrm{O}$ mesmo apresentou boa consistência de medidas e validade de conteúdo e face. Indicadores de reprodutibilidade (consistência de medidas teste-reteste) foram de moderados a altos na maioria dos itens do instrumento, sendo que os coeficientes de concordância variaram de 0,52 a 1,0.

A coleta dos dados foi realizada no período de abril a outubro de 2006, recorrendo-se a aplicação dos questionários em sala de aula, sem a presença dos professores, para todos os alunos presentes no dia da coleta, independentemente de idade. Os alunos foram continuamente assistidos pelos aplicadores (sempre dois por turma), que esclareceram e auxiliaram no preenchimento das informações.

A participação foi voluntária e todos os estudantes foram informados de que poderiam desistir em qualquer etapa da coleta de dados. Além disso, os questionários não continham qualquer tipo de identificação pessoal (nome, matrícula). Um termo de consentimento passivo foi usado para obter dos pais de estudantes com 
idade inferior a 18 anos permissão para que os mesmos participassem do estudo. O termo continha indicação clara do objetivo do estudo e solicitava que os pais comunicassem a escola caso não concordassem com a participação dos filhos. Estudantes com 18 anos de idade ou mais assinavam o próprio termo, indicando a sua concordância em participar do estudo.

Para fins de análise, foram consideradas variáveis dependentes: o nível de atividade física geral, a exposição a comportamento sedentário e a participação nas aulas de educação física. Os critérios adotados para a classificação do nível de atividade física obedeceram às recomendações de Cavill et al ${ }^{24}$, considerando-se fisicamente "ativo" o sujeito que referiu acumular, no mínimo, 60 minutos de atividades físicas diárias em cinco ou mais dias da semana. Os demais sujeitos foram classificados como “insuficientemente ativos". Conforme critério adotado em estudo prévio ${ }^{25}$, a exposição a comportamento sedentário foi determinada pelo tempo em horas despendido assistindo televisão. Sujeitos que relataram assistir habitualmente três ou mais horas de televisão por dia foram considerados expostos, sendo que duas variáveis relativas à exposição a comportamento sedentário foram consideradas nas análises, uma refletindo a conduta do adolescente nos dias da semana e outra refletindo os finais de semana. Participação nas aulas de educação física foi definida como uma variável dicotômica (participantes/não participantes), na qual foram considerados "participantes" os adolescentes que referiram participar de, pelo menos, uma aula por semana.

A afiliação e a prática religiosa (praticante/não praticante) foram as variáveis independentes. A afiliação religiosa foi determinada por uma única pergunta (“qual a sua religião?”), oferecendo-se as seguintes categorias de resposta: não tenho religião, católica, evangélica, espírita ou outra. Posteriormente, decidiu-se por agrupar "espírita" e "outra" numa única categoria, em decorrência da baixa frequência de respondentes. Em relação à prática religiosa foi utilizada também uma pergunta ("você se considera praticante da sua religião?"), sendo as repostas categorizadas dicotomicamente ( $\operatorname{sim} /$ não).

Os potenciais fatores de confusão considerados nas analises multivariáveis foram: sexo (masculino/feminino); faixa etária (14-16/17-19 anos); cor da pele (branca/não branca); turno das aulas (diurno/noturno); serie escolar (1 ${ }^{\mathrm{a}}, 2^{\mathrm{a}}$ e $3^{\mathrm{a}}$ ), porte da escola (pequeno, médio e grande), local de residência (urbano/rural), morar com os pais ( $\operatorname{sim} /$ não), escolaridade materna (baixa $=\leq 8$, média $=9-11$ e alta $=\geq 12$ anos) e situação ocupacional do adolescente (trabalhador/não trabalhador). Foi considerado trabalhador o adolescente que relatou ter emprego regular e receber salário, independente de ter ou não carteira assinada.

Para a tabulação dos dados, utilizou-se o programa Epi Data (versão 3.1). A função "Check" foi utilizada para controlar eletronicamente a entrada de dados na fase de digitação. A fim de detectar e corrigir erros, a entrada de dados foi repetida e os arquivos foram comparados. Outras informações sobre os procedimentos metodológicos do estudo poderão ser encontradas em publicações prévias ${ }^{25,26}$.

A análise dos dados foi realizada utilizando-se o pacote estatístico SPSS versão 15. Análises bivariáveis foram realizadas mediante utilização do teste de Qui-quadrado $\left(\chi^{2}\right)$ para heterogeneidade e para tendência (no caso de variáveis em escala ordinal). $\mathrm{Na}$ análise multivariada, utilizou-se regressão logística binária separadamente para cada variável dependente, considerando-se os seguintes desfechos: (1) insuficientemente ativo; (2) comportamento sedentário em dias da semana (3+ horas/dia assistindo TV); (3) comportamento sedentário em dias do final de semana (3+ horas/dia assistindo TV); e, (4) não participar das aulas de educação física. 
As análises foram realizadas em duas etapas: primeiro, efetuando-se regressões simples (não ajustadas) das variáveis independentes em relação aos desfechos focalizados no estudo. Em seguida, obedecendo a um modelo hierárquico de entrada das variáveis, procederam-se análises multivariáveis visando determinar se os indicadores de religiosidade estavam ou não associados aos desfechos. $\mathrm{O}$ modelo hierárquico considerou três níveis: a) um nível distal, onde foram incluídas as variáveis demográficas (sexo, idade e cor da pele); b) um nível intermediário, onde foram incluídos os fatores socioeconômicos e relacionados à escola (turno, série escolar, porte da escola, local de residência, morar com os pais, situação ocupacional do adolescente e escolaridade materna); e, c) o nível proximal, onde foram incluídos os fatores de religiosidade (prática e afiliação religiosas). As variáveis permaneciam no modelo quando o valor $\mathrm{p}$ identificado era inferior a 0,2 . Os resultados são apresentados na forma de razões de Odds e respectivos intervalos de confiança.

\section{RESULTADOS}

Foram visitadas 76 escolas (11\% do total de escolas estaduais do estado de Pernambuco) em 44 municípios, o que representa aproximadamente $23 \%$ do total de municípios pernambucanos. Do total de estudantes com idade entre 14 e 19 anos presentes nas escolas na ocasião da visita para coleta de dados, 83 se recusaram a participar do estudo (1,9\% de recusas), assim, responderam ao questionário um total de 4.207 estudantes (média de 16,8 anos; s =1,4), dos quais 59,8\% eram do sexo feminino.

Com este quantitativo de participantes $(n=4207)$, verificou-se que seria possível detectar como significativos valores de Odds Ratio (OR) iguais ou superiores a 1,2 , considerando-se intervalo de confiança de $95 \%$, poder de $80 \%$ e prevalência do desfecho de $35 \%$ entre não expostos e de 39\% entre expostos. Na Tabela 1 estão apresentadas as características demográficas, socioeconômicas, relacionadas à escola e indicadores de religiosidade dos adolescentes estratificados por sexo.

A prevalência de níveis insuficientes de prática de atividade física foi de 65,1\% (IC95\%: 63,6-66,6), sendo significativamente $(\mathrm{p}<0,001)$ maior entre as moças (70,2\%; IC95\%: 68,4-71,9) em comparação aos rapazes (57,7\%; IC95\%: 55,3-60,0). Verificou-se ainda que 64,9\% (IC95\%: 63,4-66,3) dos respondentes relataram não participar das aulas de educação física, sendo que a proporção de sujeitos que relatou não participar foi significativamente maior entre as moças (67,8\%; IC95\%:66,0-69,6) que entre os rapazes (60,5\%; IC95\%:58,2-62,8).

Observou-se que em dias de semana 40,8\% dos sujeitos relataram assistir 3 ou mais horas de televisão por dia, conduta definida operacionalmente neste estudo como comportamento sedentário. Não foram identificadas diferenças significativas entre rapazes e moças. Em relação à conduta dos adolescentes nos dias de final de semana, notou-se que a prevalência de comportamento sedentário foi superior ao observado em dias de semana (49,9\%; IC95\%:48,4-51,4) e significativamente maior entre os rapazes $(54,0 \%$; IC95\%: 51,6-56,4) em comparação às moças (47,1\%; IC95\%: 45,2-49,1).

As análises bivariáveis evidenciaram que a afiliação religiosa é um fator associado aos quatro desfechos considerados neste estudo: nível insuficiente de atividade física, não participação nas aulas de educação física, comportamento sedentário em dias da semana e comportamento sedentário em dias do final de semana (Figura 1). Foi observado que a prevalência de nível insuficiente de atividade física foi maior entre os adolescentes que referiram ser "católicos", por outro lado, também 
Tabela 1 - Características das variáveis demográficas, socioeconômicas e religiosas de escolares conforme o sexo. Pernambuco, Brasil, 2006.

\begin{tabular}{|c|c|c|c|c|c|c|c|}
\hline \multirow{2}{*}{ Variável } & \multirow{2}{*}{ Categoria } & \multicolumn{2}{|c|}{ Rapazes } & \multicolumn{2}{|c|}{ Moças } & \multicolumn{2}{|c|}{ Todos } \\
\hline & & $\mathrm{n}$ & $\%$ & $n$ & $\%$ & $\mathrm{n}^{*}$ & $\%$ \\
\hline \multirow{2}{*}{ Turno } & Diurno & 908 & 53,9 & 1.506 & 60,0 & 2.414 & 57,6 \\
\hline & Noturno & 778 & 46,1 & 1.002 & 40,0 & 1.780 & 42,4 \\
\hline \multirow{3}{*}{ Série } & $1^{\circ}$ ano & 778 & 46,1 & 1.099 & 43,8 & 1.877 & 44,7 \\
\hline & $2^{\circ}$ ano & 522 & 31,0 & 818 & 32,6 & 1.340 & 31,9 \\
\hline & $3^{\circ}$ ano & 386 & 22,9 & 593 & 23,6 & 979 & 23,3 \\
\hline \multirow{2}{*}{ Faixa etária } & $14-16$ & 598 & 35,4 & 1.165 & 46,4 & 1.763 & 42,0 \\
\hline & $17-19$ & 1.089 & 64,6 & 1.346 & 53,6 & 2.435 & 58,0 \\
\hline \multirow{2}{*}{ Cor da pele } & Branca & 417 & 24,8 & 639 & 25,5 & 1.056 & 25,2 \\
\hline & Outra & 1.262 & 75,2 & 1.866 & 74,5 & 3.128 & 74,8 \\
\hline \multirow{2}{*}{ Local de residência } & Urbana & 1.311 & 78,1 & 1.983 & 79,5 & 3.294 & 79,0 \\
\hline & Rural & 367 & 21,9 & 510 & 20,5 & 877 & 21,0 \\
\hline \multirow{3}{*}{ Escolaridade materna } & Baixa & 1.086 & 69,4 & 1.771 & 74,5 & 2.857 & 72,5 \\
\hline & Média & 352 & 22,5 & 480 & 20,2 & 832 & 21,1 \\
\hline & Alta & 127 & 8,1 & 126 & 5,3 & 253 & 6,4 \\
\hline \multirow{2}{*}{ Mora com os pais } & Sim & 1.130 & 68,1 & 1.510 & 60,6 & 2.640 & 63,6 \\
\hline & Não & 529 & 31,9 & 981 & 39,4 & 1.510 & 36,4 \\
\hline \multirow{2}{*}{ Situação ocupacional } & Não trabalha & 1.157 & 69,2 & 2.119 & 84,8 & 3.276 & 78,5 \\
\hline & Trabalha & 514 & 30,8 & 381 & 15,2 & 895 & 21,5 \\
\hline \multirow{3}{*}{ Porte da escola } & $<200$ & 152 & 9,0 & 221 & 8,8 & 373 & 8,9 \\
\hline & $200-499$ & 456 & 27,0 & 628 & 25,0 & 1.084 & 25,8 \\
\hline & $>500$ & 1.079 & 64,0 & 1.662 & 66,2 & 2.741 & 65,3 \\
\hline \multirow{4}{*}{ Afiliação religiosa } & Não tem & 325 & 19,4 & 281 & 11,2 & 606 & 14,5 \\
\hline & Católico & 926 & 55,2 & 1.541 & 61,6 & 2.467 & 59,0 \\
\hline & Evangélico & 358 & 21,3 & 600 & 24,0 & 958 & 22,9 \\
\hline & Outra & 70 & 4,1 & 80 & 3,2 & 150 & 3,6 \\
\hline \multirow{2}{*}{ Praticante da religião } & Sim & 965 & 57,7 & 1.770 & 71,1 & 2.735 & 65,7 \\
\hline & Não & 708 & 42,3 & 721 & 28,9 & 1.429 & 34,3 \\
\hline
\end{tabular}

* o número de respondentes difere de 4207 em virtude de dados inexistentes (missing values) para algumas variáveis.

foi entre estes que se encontrou menor prevalência de não participação nas aulas de educação física. Em relação ao comportamento sedentário, a prevalência foi inferior entre os adolescentes "evangélicos" tanto em dias de semana quanto em dias do final de semana. Ao recorrer à estratificação das análises por sexo, verificouse entre os rapazes que estas associações não eram significativas, exceto em relação ao comportamento sedentário em dias do final de semana.

Ainda considerando os resultados das análises bivariáveis, identificou-se que a prática religiosa é um fator associado a não participação nas aulas de educação física, comportamento sedentário em dias da semana e comportamento sedentário em dias do final de semana. Os resultados apontam para prevalências mais elevadas destes desfechos entre os adolescentes que referiram não serem praticantes de uma religião (Figura 2).

As análises de regressão logística com ajustamento para potenciais fatores de confusão permitiram identificar que tanto a afiliação religiosa quanto a prática religiosa são fatores associados ao nível de atividade física, participação nas aulas de educação física e comportamento sedentário. Entretanto, o sentido das associações parece diferir, enquanto a prática religiosa está inversamente associada, observou-se que ter uma determinada afiliação religiosa está diretamente associado à ocorrência de três dos quatro 

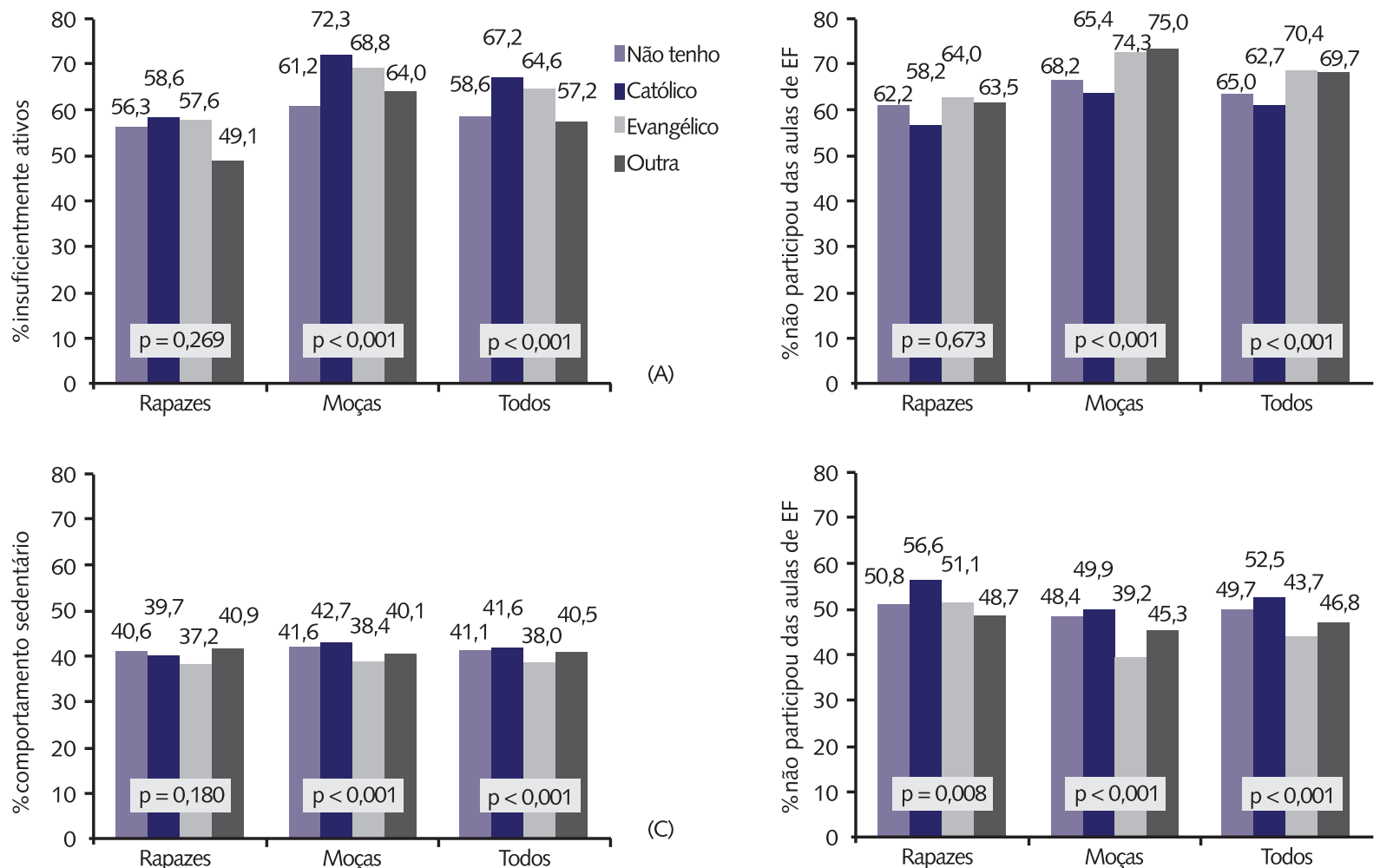

Figura 1 - Frequência absoluta e relativa de adolescentes expostos a nível insuficiente de atividade física (A), não participação nas aulas de educação física (B), comportamento sedentário em dias de semana (C) e comportamento sedentário em dias do final de semana (D) segundo afiliação religiosa, por sexo. Pernambuco, Brasil, 2006.

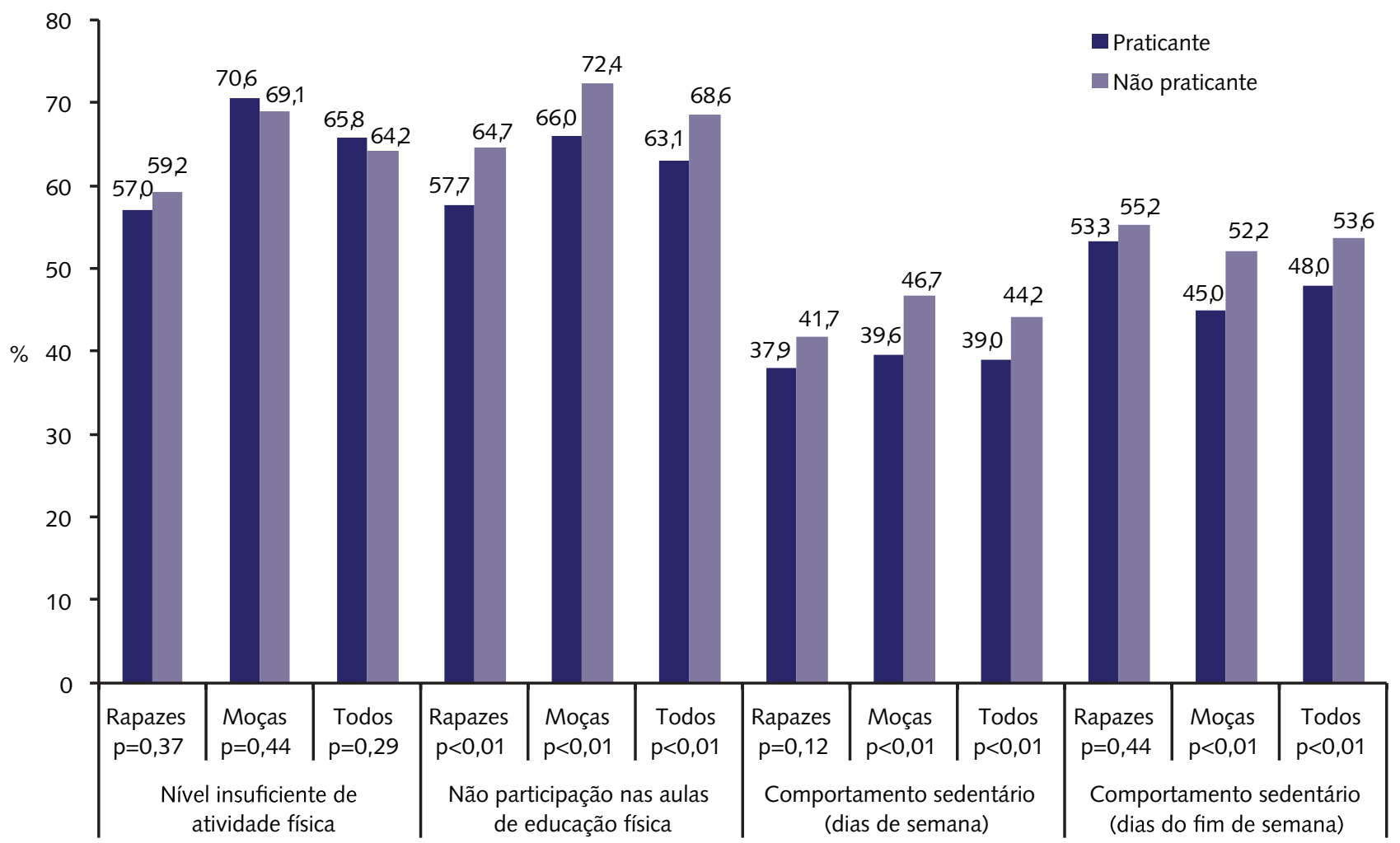

Figura 2 - Frequência absoluta e relativa de adolescentes expostos a nível insuficiente de atividade física, não participação nas aulas de educação física, comportamento sedentário em dias de semana e comportamento sedentário em dias do final de semana segundo prática religiosa, por sexo. Pernambuco, Brasil, 2006. 
desfechos analisados no presente estudo (Tabela 2). Adolescentes que referiram ser católicos apresentaram chance 39\% e 26\% superior de apresentaram, respectivamente, nível insuficiente de prática de atividade física e comportamento sedentário em dias do final de semana em comparação aos adolescentes que informaram não ter uma afiliação religiosa. Por outro lado, identificou-se que os adolescentes evangélicos em comparação aos que informaram não ter uma afiliação religiosa apresentaram chance $76 \%$ superior de não participar das aulas de educação física.

Tabela 2 - Análises de regressão logística bruta e ajustada da associação entre indicadores de religiosidade (afiliação e prática religiosa) e níveis insuficientes de atividade física (desfecho 1), não participação nas aulas de educação física (desfecho 2) e comportamento sedentário em dias de semana (desfecho 3) e do final de semana (desfecho 4). Pernambuco, Brasil, 2006.

\begin{tabular}{|c|c|c|c|c|c|c|c|}
\hline Variável & Categoria & OR bruto & IC $95 \%$ & valor $\mathrm{p}$ & OR ajustado & IC $95 \%$ & valor $\mathrm{p}$ \\
\hline \multicolumn{8}{|c|}{ Desfecho 1: níveis insuficientes de atividade física } \\
\hline \multirow{4}{*}{ Afiliação religiosa } & Não tenho & 1 & & & 1 & & \\
\hline & Católica & 1,45 & $1,21-1,73$ & $<0,01$ & 1,39 & $1,14-1,71$ & $<0,01$ \\
\hline & Evangélica & 1,29 & $1,05-1,59$ & 0,016 & 1,22 & $0,97-1,55$ & 0,095 \\
\hline & Outros & 1,16 & $0,82-1,66$ & 0,403 & 1,23 & $0,84-1,79$ & 0,281 \\
\hline \multirow{2}{*}{ Prática religiosa } & Não & 1 & & & 1 & & \\
\hline & Sim & 1,08 & $0,94-1,23$ & 0,291 & 0,91 & $0,78-1,06$ & 0,239 \\
\hline \multicolumn{8}{|c|}{ Desfecho 2: não participação nas aulas de educação física } \\
\hline \multirow{4}{*}{ Afiliação religiosa } & Não tenho & 1 & & & 1 & & \\
\hline & Católica & 0,91 & $0,75-1,09$ & 0,312 & 1,17 & $0,94-1,45$ & 0,167 \\
\hline & Evangélica & 1,28 & $1,03-1,59$ & 0,026 & 1,76 & $1,36-2,27$ & $<0,01$ \\
\hline & Outros & 0,99 & $0,69-1,42$ & 0,926 & 1,35 & $0,91-2,01$ & 0,138 \\
\hline \multirow{2}{*}{ Prática religiosa } & Não & 1 & & & 1 & & \\
\hline & Sim & 0,78 & $0,68-0,90$ & $<0,01$ & 0,70 & $0,60-0,83$ & $<0,01$ \\
\hline \multicolumn{8}{|c|}{ Desfecho 3: comportamento sedentário em dias de semana } \\
\hline \multirow{4}{*}{ Afiliação religiosa } & Não tenho & 1 & & & 1 & & \\
\hline & Católica & 1,20 & $0,85-1,22$ & 0,829 & 1,18 & $0,96-1,44$ & 0,117 \\
\hline & Evangélica & 0,88 & $0,71-1,08$ & 0,222 & 0,99 & $0,79-1,26$ & 0,965 \\
\hline & Outros & 1,21 & $0,85-1,71$ & 0,286 & 1,32 & $0,91-1,90$ & 0,141 \\
\hline \multirow{2}{*}{ Prática religiosa } & Não & 1 & & & 1 & & \\
\hline & Sim & 0,81 & $0,71-0,92$ & $<0,01$ & 0,79 & $0,69-0,92$ & $<0,01$ \\
\hline \multicolumn{8}{|c|}{ Desfecho 4: comportamento sedentário em dias do final de semana } \\
\hline \multirow{4}{*}{ Afiliação religiosa } & Não tenho & 1 & & & 1 & & \\
\hline & Católica & 1,12 & $0,94-1,34$ & 0,221 & 1,26 & $1,03-1,53$ & 0,024 \\
\hline & Evangélica & 0,79 & $0,64-0,97$ & 0,022 & 0,91 & $0,72-1,14$ & 0,407 \\
\hline & Outros & 0,94 & $0,68-1,33$ & 0,734 & 0,99 & $0,69-1,44$ & 0,995 \\
\hline \multirow{2}{*}{ Prática religiosa } & Não & 1 & & & 1 & & \\
\hline & Sim & 0,80 & $0,70-0,91$ & $<0,01$ & 0,79 & $0,68-0,91$ & $<0,01$ \\
\hline
\end{tabular}

\section{DISCUSSÃO}

O objetivo deste estudo foi analisar a associação de indicadores de religiosidade (afiliação e prática religiosa) com o nível de atividade física, comportamento sedentário e participação em aulas de educação física em adolescentes estudantes. Os resultados evidenciaram que existe associação entre os indicadores de religiosidade e os desfechos analisados. Em relação à afiliação religiosa, observouse que: (1) adolescentes católicos em comparação com àqueles que referiram não ter religião apresentaram maior chance de serem insuficientemente ativos e de estarem expostos a comportamento sedentário em dias do final de semana; e (2) 
adolescentes evangélicos apresentaram maior chance de não participação nas aulas de educação física. Por fim, pode-se sintetizar ainda que a prática religiosa é um fator que não está associado ao nível de atividade física, mas diminui a chance de não participação nas aulas de educação física e de exposição a comportamento sedentário, tanto em dias de semana quanto do final de semana.

É importante interpretar com cautela os resultados apresentados neste estudo, principalmente no sentido de evitar inferências causais entre a religiosidade e as condutas de saúde focalizadas, principalmente porque o comportamento em relação às atividades físicas e a exposição a comportamentos sedentários tem uma cadeia causal complexa ${ }^{27}$ e não pode ser atribuído exclusivamente à religiosidade. Além disso, no presente estudo não se pode descartar a possibilidade de viés de informação, pois mesmo com garantia de anonimato é possível que alguns alunos tenham omitido a afiliação religiosa por autocensura ou erro de memória. Uma estratégia adotada para atenuar esta limitação foi a utilização de um questionário que foi previamente testado e que apresentou bom nível de reprodutibilidade, com coleta de dados realizada por entrevistadores treinados e experientes na operação deste tipo de trabalho de campo.

Este estudo foi conduzido com uma amostra relativamente grande, representativa dos estudantes do ensino médio da rede pública, no Estado de Pernambuco. Adicionalmente, o tamanho amostral era suficientemente grande para garantir adequado poder estatístico para análises que foram realizadas.

Por fim, até onde se tem conhecimento esta é uma das primeiras investigações abrangentes realizadas no Brasil com o intuito de analisar a associação entre a religiosidade e a prática de atividade física e comportamento sedentário. Em muitos dos estudos sobre fatores associados a condutas de saúde em adolescentes o foco recaiu principalmente na investigação de como a religiosidade pode estar associada a consumo pesado de álcool e tabaco ${ }^{28,29}$. Além de focalizar na análise de condutas de saúde pouco exploradas, no presente estudo foram considerados simultaneamente dois fatores da religiosidade (a afiliação e a prática religiosa), quando, usualmente, cada um destes fatores é analisado isoladamente ${ }^{13}$.

Apesar de ter sido realizado com um grupo relativamente pequeno de sujeitos, de ter selecionado os sujeitos em uma única escola e ter usado diferentes indicadores de religiosidade, o estudo de Loch $^{15}$ apresentou evidências convergentes em relação ao observado no presente estudo. Segundo $\operatorname{Loch}^{15}$, a "participação em grupos de jovens" e a frequência de participação em eventos religiosos foram fatores positivamente associados a um maior nível de prática de atividade física entre os adolescentes investigados.

Resultados semelhantes também foram observados em estudos realizados com adultos estadunidenses. Estes indicaram que "compromisso religioso" (entre as mulheres) e "apoio social divino" (entre os homens) foram indicadores de religiosidade positivamente associados ao nível de atividade física dos sujeitos ${ }^{23}$. Similarmente, investigação realizada com cerca de seis mil adultos permitiu constatar que as pessoas que participavam de eventos religiosos pelo menos uma vez por semana apresentavam maiores níveis de atividade física ${ }^{22}$. Desta forma, a religiosidade é apresentada como um facilitador para a adoção de um estilo de vida fisicamente ativo ${ }^{30}$.

Entretanto, um achado específico do presente estudo alerta para o fato de que alguns elementos de religiosidade, por exemplo, a afiliação religiosa podem atuar como barreira para prática de atividades físicas e facilitador da exposição a comportamento sedentário, como o que foi observado entre os adolescentes 
que referiram ser "católicos". Estes sujeitos apresentariam, portanto, chance mais elevada de adotarem uma condição combinada de risco à saúde: baixo nível de atividade física aliado a comportamento sedentário. No entanto, ainda não se dispõe de evidências consistentes que possam explicar o mecanismo de influência da afiliação religiosa na exposição a comportamentos sedentários.

Ainda em relação à afiliação religiosa, também não há dados suficientes para explicar a maior chance de não participação nas aulas de educação física entre os adolescentes evangélicos em comparação àqueles que relataram não ter religião. É possível que a doutrina adotada por algumas denominações evangélicas não estimule ou valorize a participação nas aulas por não se reconhecer a importância deste componente curricular para formação pessoal, humanística e espiritual dos sujeitos. Mas estas ilações precisam ser mais bem investigadas, possivelmente mediante abordagens qualitativas de investigação.

Os achados relatados nesta pesquisa reforçam evidências apresentadas em outros estudos de que a religiosidade é um fator associado à prática de atividade física ${ }^{13,15}$ e comportamento sedentário ${ }^{14}$. Porém, não foram encontradas pesquisas que evidenciassem esta associação com a participação nas aulas de educação física. Alguns fatores parecem estar diretamente associados enquanto outros apresentam relação inversa com tais condutas. A quantidade de estudos disponíveis e a qualidade do delineamento adotado impede que generalizações possam ser efetuadas neste momento, mas é possível supor que a investigação nesta linha de pesquisa possa revelar estratégias de intervenção para promoção da atividade física a partir de fatores religiosos.

\section{Financiamento}

Estudo apoiado com auxílio financeiro da Coordenação de Aperfeiçoamento de Pessoal de Nível Superior, do Conselho Nacional de Desenvolvimento Científico e Tecnológico e da Fundação de Amparo à Ciência e Tecnologia do Estado de Pernambuco.

\section{REFERÊNCIAS}

1. United States Department of Health and Human Services. Physical Activity Guidelines Advisory Committee Report, 2008. Washington DC: United States Department of Health and Human Services; 2008.

2. Tassitano RM, Bezerra J, Tenorio MCM, Colares V, Barros MVG, Hallal PC. Atividade física em adolescentes brasileiros: uma revisão sistemática. Rev Bras Cineantropom Desempenho Hum. 2007;9(1):55-60.

3. Colley RC, Garrighet D, Janssen I, Craig CL, Clarke J, Tremblay Ms. Physical activity of Canadian children and youth: accelerometer results from the 2007 to 2009 Canadian Health Measures Survey. Health Rep. 2011; 22(1):15-23.

4. Bastos JP, Araújo CLP, Hallal PC. Prevalence of insufficient physical activity and associated factors in Brazilian adolescents. J Phys Act Health. 2008;5(6):777-94.

5. Van Der Horst K, Paw MJ, Twisk JW, Van Mechelen W. A brief review on correlates of physical activity and sedentariness in youth. Med Sci Sports Exerc. 2007;39(8):1241-50.

6. Allison KR, Adlaf EM, Dwyer JJ, Lysy DC, Irving HM. The decline in physical activity among adolescent students: a cross-national comparison. Can J Public Health. 2007; 98(2):97-100.

7. Brodersen NH, Steptoe A, Boniface DR, Wardle J. Trends in physical activity and sedentary behavior in adolescence: ethnic and socioeconomic differences. Br J Sports Med. 2007; 41(3):140-4.

8. Hallal PC, Knuth AG, Cruz DKA, Mendes MI, Malta DC. Prática de atividade física em adolescentes brasileiros. Ciênc Saúde Coletiva. 2010;15(2):3035-42. 
9. Martinez-Gomez D, Rey-López JP, Chillón P, Gómez-Martínez S, Vicente-Rodríguez $\mathrm{G}$, et al. Excessive TV viewing and cardiovascular disease risk factors in adolescents. The AVENA cross-sectional study. BMC Public Health. 2010;10:274.

10. Bibbins-Domingo K, Coxson P, Pletcher MJ, Lightwood J, Goldman L. Adolescent overweight and future adult coronary heart disease. N Engl J Med. 2007;357(23): 2371-9.

11. Mckenzie TL, Nader PR, Strikmiller PK, Yang M, Stone EJ, et al. School Physical Education: Effect of the Child and Adolescent Trial for Cardiovascular Health. Prev Med. 1996;25(4):423-31.

12. Cox AE, Smith AL, Williams L. Change in physical education motivation and physical activity behavior during middle school. J Adolesc Health. 2008;43(5):506-13.

13. Rew L, Wong YJ. A systematic review of associations among religiosity/spirituality and adolescent health attitudes and behaviors. J Adolesc Health. 2006;38(4):433-42.

14. Kahan D. Relationships among religiosity, physical activity, and sedentary behavior in jewish adolescents. Pediatr Exerc Sci. 2004;16(1):54-63.

15. Loch MR. Comportamentos relacionados à saúde e indicadores de religiosidade em adolescentes escolares [dissertação de mestrado]. Florianópolis: Universidade Federal de Santa Catarina; 2006.

16. Harrison MO, Edwards CL, Koening HG, Bosworth HB, Decastro L, Wood M. Religiosity/ spirituality and pain in patients with sickle cell disease.J Nerv Ment Dis. 2005;193(4):250-7.

17. Moreira-Almeida A, Neto FL, Koenig HG. Religiousness and mental health: a review. Rev Bras Psiquiatr. 2006;28(3):242-50.

18. Williams DR, Sternthal MJ. Spirituality, religion and health: evidence and research directions. Med J Aust. 2007;186(10 suppl):S47-50.

19. Guimarães HP, Avezum A. O impacto da espiritualidade na saúde física. Rev Psiquiatr Clín. 2007;34(1 supl):88-94.

20. Kendler KS, Gardner CO, Prescott CA. Religion, psychopathology, and substance use and abuse; a multimeasure, genetic-epidemiologic study. Am J Psychiatry. 1997;154(3):322-9.

21. Miller L, Gur M. Religiousness and sexual responsibility in adolescents girls. J Adolesc Health. 2002;31(5):401-6.

22. Merril RM, Thygerson AL. Religious preference, church activity and physical exercise. Prev Med. 2001;33(1):38-45.

23. Kim KH, Sobal J. Religion, social support, fat intake and physical activity. Public Health Nutr. 2004;7(6):773-81.

24. Cavill N, Biddle S, Sallis JF. Health enhancing physical activity for young people: statement of the United Kingdom expert consensus conference. Pediatr Exerc Sci. 2001;1(1):12-25.

25. Tenório MCM, Barros MVG, Tassitano RM, Bezerra J, Tenório JM, Hallal PC. Atividade física e comportamento sedentário em adolescentes estudantes do ensino médio. Rev Bras Epidemiol. 2010;13(1):105-17.

26. Santos CM, Wanderley Júnior, Barros SSH, Farias Júnior JC, Barros MVG. Prevalência e fatores associados à inatividade física nos deslocamentos para escola em adolescentes. Cad Saúde Pública. 2010;26(7):1419-30.

27. Dumith SC. Proposta de um modelo teórico para adoção da prática de atividade física. Rev Bras Ativ Fís Saúde. 2008;13(2):110-20.

28. Tavares BF, Béria JU, Lima MS. Fatores associados ao uso de drogas entre adolescentes escolares. Rev Saúde Pública. 2004;38(6):787-96.

29. Souza DPO, Silveira Filho DX. Uso recente de álcool, tabaco e outras drogas entre estudantes adolescentes trabalhadores e não trabalhadores. Rev Bras Epidemiol. 2007;10(2):276-87.

30. Shuval K, Weissblueth E, Araida A, Brezis M, Faridi Z, Ali A, et al. The role of culture, environment, and religion in the promotion of physical activity among Arab Israelis. Prev Chronic Dis. 2008;5(3):A88.

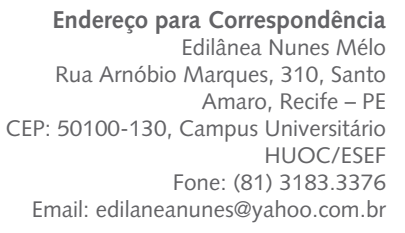

Recebido $01 / 10 / 2012$

Revisado 29/11/2012 Aprovado 03/12/2012 